\title{
Classification of discrete modular symmetries in type IIB flux vacua
}

\author{
Tatsuo Kobayashi ${ }^{1, *}$ and Hajime Otsuka ${ }^{2, \dagger}$ \\ ${ }^{1}$ Department of Physics, Hokkaido University, Sapporo 060-0810, Japan \\ ${ }^{2}$ KEK Theory Center, Institute of Particle and Nuclear Studies, KEK, 1-1 Oho, \\ Tsukuba, Ibaraki 305-0801, Japan
}

(Received 6 April 2020; accepted 28 April 2020; published 14 May 2020)

\begin{abstract}
We classify discrete modular symmetries in the effective action of Type IIB string on toroidal orientifolds with three-form fluxes, emphasizing on $T^{6} / \mathbb{Z}_{2}$ and $T^{6} /\left(\mathbb{Z}_{2} \times \mathbb{Z}_{2}^{\prime}\right)$ orientifold backgrounds. In the threeform flux background, the modular group is spontaneously broken down to its congruence subgroup whose pattern is severely constrained by a quantization of fluxes and tadpole cancellation conditions. We explicitly demonstrate that the congruence subgroups appearing in the effective action arise on magnetized D-branes wrapping certain cycles of tori.
\end{abstract}

DOI: 10.1103/PhysRevD.101.106017

\section{INTRODUCTION}

In the low-energy effective action of higher-dimensional theory, moduli are ubiquitous fields and they have certain symmetries, originating from the higher-dimensional gauge and/or Lorentz symmetries. As an example, axionlike fields have the so-called shift symmetries associated with the gauge symmetries of higher-form fields, which play an important role in solving the strong CP [1] and hierarchy problems [2], the candidates of dark matter [3-5], and inflaton [6-8]. Such shift symmetries are useful to control the action against the higher-order corrections.

Torus and orbifold compactifications have the modular symmetry. For example, the two-dimensional tori and orbifolds have the modular symmetry, $S L(2, \mathbb{Z})$. Modular symmetries in effective supergravity theory have been studied, e.g., for moduli stabilization, supersymmetry breaking [9,10], and inflation models [11]. Moreover, Yukawa couplings and higher-order couplings depend on moduli. (See for moduli-dependent couplings in heterotic orbifold models [12], intersecting D-brane models [13], and magnetized D-brane models [14].) They transform nontrivially under the modular symmetry. Then, Yukawa matrices transform nontrivially, and the modular group transforms flavors nontrivially, that is, the flavor symmetry. Indeed, matter fields on these compactifications transform each other under the modular group. For example, a finite

\footnotetext{
kobayashi@particle.sci.hokudai.ac.jp

thotsuka@post.kek.jp
}

Published by the American Physical Society under the terms of the Creative Commons Attribution 4.0 International license. Further distribution of this work must maintain attribution to the author(s) and the published article's title, journal citation, and DOI. Funded by SCOAP. number of zero modes appear on torus and orbifold compactifications with magnetic fluxes. These zero modes transform under the modular group. That is, these zero modes become finite representations of the modular group or its subgroups, which are finite discrete groups $[15,16] .{ }^{1}$

Recently, discrete subgroups arising from the $S L(2, \mathbb{Z})$ modular group as well as its congruence subgroups have been utilized for the flavor symmetry of quarks/leptons, including $C P$ violation and applications for leptogenesis and dark matter candidates [22-58]. The reason to attract attention of researchers is that the quotients $\Gamma_{N}=$ $S L(2, \mathbb{Z}) / \Gamma(N)$ by congruence subgroups $\Gamma(N)$ correspond to the well-known discrete finite groups such as $\Gamma_{2} \simeq S_{3}, \Gamma_{3} \simeq A_{4}, \Gamma_{4} \simeq S_{4}, \Gamma_{5} \simeq A_{5}$, which provide interesting flavor structures of quarks and/or leptons [59].

The three-form fluxes can stabilize some or all of the complex structure moduli [60]. Then, the geometrical symmetries of $T^{6}=T_{1}^{2} \times T_{2}^{2} \times T_{3}^{2}$ with nonvanishing three-form fluxes can be different from $\bigotimes_{i=1}^{3} S L(2, \mathbb{Z})_{i}$. They would provide us with new possibilities for starting points toward the above studies of particle physics and cosmologies.

In this paper, we study a simple type IIB flux compactification on toroidal orientifolds with and without D-branes, where the modular symmetries associated with tori are partially broken into subgroups by the three-form fluxes. Subgroups of the modular group $\otimes_{i=1}^{3} S L(2, \mathbb{Z})_{i}$ emerge in the flat directions of moduli fields such that the modular transformation is viable in the low-energy effective action. Our aim is to classify the modular groups and its subgroups in the effective action if it exists, meaning that we do not consider the stabilization of the remaining massless moduli

\footnotetext{
${ }^{1}$ See also for recent relevant works in magnetized D-brane models [17-19] and heterotic orbifold models [20,21].
} 
in this work. Such an approach is similar to the recent swampland program [61-63] where the allowable moduli space of massless modes is taken into account.

This paper is organized as follows. In Sec. II, we first show the realization of the modular symmetry and its breaking in the low-energy effective action of type IIB string theory on a factorizable six-torus subject to $\mathbb{Z}_{2}$ identification, i.e., $T^{6} / \mathbb{Z}_{2}$. The idea to obtain the congruence subgroups of the modular group is basically given in [64]. They focus on the flat axionic direction which can be enlarged to the Planckian field region by an existence of fluxes. In this paper, we extend their analysis and classify the remaining modular symmetry in the low-energy effective action. Next, we move on to $T^{6} /\left(\mathbb{Z}_{2} \times \mathbb{Z}_{2}^{\prime}\right)$ orientifold background where several semirealistic models are proposed in [65-68]. In Sec. III, we classify the breaking pattern of the modular group on $T^{6} /\left(\mathbb{Z}_{2} \times \mathbb{Z}_{2}^{\prime}\right)$ orientifold with magnetized D-branes wrapping certain cycles of tori. Similar to the analysis in Sec. II, we enumerate the possible congruence subgroups in a concrete three-generation model. It turns out that the remaining modular symmetry in the effective action is severely constrained by the quantization of fluxes and tadpole cancellation conditions. Section IV is devoted to the conclusions and discussions. In the Appendix, we show our conventions of congruence subgroups.

\section{MODULAR SYMMETRY ON $T^{6} / \mathbb{Z}_{2}$ TOROIDAL ORIENTIFOLD}

In this section, we briefly review the modular symmetry in four-dimensional (4D) effective action of type IIB string on $T^{6} / \mathbb{Z}_{2}$ toroidal orientifold with three-form fluxes. After demonstrating the breaking mechanism of the modular symmetry discussed in [64], we extend their analysis and classify patterns of congruence subgroups in the lowenergy effective action.

\section{A. Effective action}

The setup is the $4 \mathrm{D}$ effective action of type IIB string on toroidal orientifold, in particular the factorizable six-torus $T^{6} / \mathbb{Z}_{2}=\left(T_{1}^{2} \times T_{2}^{2} \times T_{3}^{2}\right) / \mathbb{Z}_{2}$. The effective action of the closed string moduli on this background is described in the 4D $\mathcal{N}=1$ language, namely, the Kähler potential $K$ and the superpotential $W,^{2}$

$$
\begin{aligned}
K= & -\ln (-i(\tau-\bar{\tau}))-2 \ln \mathcal{V} \\
& -\ln \left(i\left(\tau_{1}-\bar{\tau}_{1}\right)\left(\tau_{2}-\bar{\tau}_{2}\right)\left(\tau_{3}-\bar{\tau}_{3}\right)\right),
\end{aligned}
$$

where $\tau=C_{0}+i e^{-\phi}$ is the axiodilaton, $\tau_{i}$ with $i=1,2,3$ are three complex structure moduli on $T_{i}^{2}$, and $\mathcal{V}$ denotes the volume of torus. The superpotential is generated by background three-form fluxes $G_{3}=F_{3}-\tau H_{3}$ [70],

$$
W=\frac{1}{l_{s}^{2}} \int \Omega \wedge G_{3},
$$

where $l_{s}=2 \pi \sqrt{\alpha^{\prime}}$ and a holomorphic three-form $\Omega$ is specified by the six real coordinates on $T^{6}\left(x^{i}, y^{i}\right)$ with $i=1,2,3$ and the complex structure moduli $\tau^{i}$,

$$
\Omega=d z_{1} \wedge d z_{2} \wedge d z_{3},
$$

with $d z^{i}=d x^{i}+\tau^{i} d y^{i}$. In a similar way, $G_{3}$ can be expanded on the basis of $H^{3}\left(T^{6}, 2 \mathbb{Z}\right),\left(\alpha_{I}, \beta^{J}\right)$, satisfying $\int_{T^{6}} \alpha_{I} \wedge \beta^{J}=\delta_{I}^{J}$,

$$
\begin{aligned}
& \frac{1}{l_{s}^{2}} F_{3}=a^{0} \alpha_{0}+a^{i} \alpha_{i}+b_{i} \beta^{i}+b_{0} \beta^{0}, \\
& \frac{1}{l_{s}^{2}} H_{3}=c^{0} \alpha_{0}+c^{j} \alpha_{i}+d_{i} \beta^{i}+d_{0} \beta^{0},
\end{aligned}
$$

where $a^{0,1,2,3}, b_{0,1,2,3}$ and $c^{0,1,2,3}, d_{0,1,2,3}$ are quantized to be even integers according to

$$
\frac{1}{l_{s}^{2}} \int F_{3} \in 2 \mathbb{Z}, \quad \frac{1}{l_{s}^{2}} \int H_{3} \in 2 \mathbb{Z} .
$$

In this paper, we restrict ourselves to consider even integers; otherwise, exotic O3-plane contributions are necessary in the system $[71,72]$. The basis of three form is explicitly given by

$$
\begin{aligned}
& \alpha_{0}=d x^{1} \wedge d x^{2} \wedge d x^{3}, \quad \alpha_{i}=\frac{1}{2} \epsilon_{i l m} d x^{l} \wedge d x^{m} \wedge d y^{i} \quad(1 \leq i \leq 3), \\
& \beta^{0}=d y^{1} \wedge d y^{2} \wedge d y^{3}, \quad \beta^{i}=-\frac{1}{2} \epsilon_{i l m} d y^{l} \wedge d y^{m} \wedge d x^{i}(1 \leq i \leq 3) .
\end{aligned}
$$

Then, we can explicitly write down the superpotential,

$$
W=\left(a^{0}-\tau c^{0}\right) \tau_{1} \tau_{2} \tau_{3}-\left(a^{1}-\tau c^{1}\right) \tau_{2} \tau_{3}-\left(a^{2}-\tau c^{2}\right) \tau_{1} \tau_{3}-\left(a^{3}-\tau c^{3}\right) \tau_{1} \tau_{2}-\sum_{i=1}^{3}\left(b_{i}-\tau d_{i}\right) \tau_{i}-\left(b_{0}-\tau d_{0}\right) .
$$

\footnotetext{
${ }^{2}$ We follow the notation of [69] and adopt the reduced Planck mass unit $M_{\mathrm{Pl}}=1$.
} 
Note that three-form fluxes induce the D3-brane charge $N_{\text {flux }}=\int_{T^{6}} H_{3} \wedge F_{3}$ which appears in the cancellation condition of D3-brane charge,

$$
N_{\mathrm{D} 3}+\frac{1}{2} N_{\mathrm{flux}}=\frac{1}{4} N_{\mathrm{O} 3}=16 .
$$

Here we employ the fact that there exist $64 \mathrm{O} 3$ planes associated with 64 fixed points on $T^{6} / \mathbb{Z}_{2}$ orientifold background. Thus, the above tadpole cancellation condition is simplified as

$c^{0} b_{0}-d_{0} a^{0}+\sum_{i}\left(c^{i} b_{i}-d_{i} a^{i}\right)=2\left(16-N_{\mathrm{D} 3}\right) \leq 32$,

where we do not consider the presence of anti-D3 branes to preserve the supersymmetry.

\section{B. Modular symmetry of the effective action}

Before we explain the mechanism to derive the discrete modular groups, we briefly review the modular symmetry of the effective action, following [64]. The effective Kähler potential (1) and superpotential (2) are invariant under $S L(2, \mathbb{Z})_{\tau}$, where $S L(2, \mathbb{Z})_{\tau}$ denotes the modular group associated with axiodilaton. The explicit modular transformations for the axiodilaton $\tau$ and the pair of three-form fluxes $\left(F_{3}, H_{3}\right)$ are given by

$\tau^{\prime}=R(\tau)=\frac{p \tau+q}{s \tau+t},\left(\begin{array}{l}F_{3}^{\prime} \\ H_{3}^{\prime}\end{array}\right)=\left(\begin{array}{ll}q & p \\ t & s\end{array}\right)\left(\begin{array}{l}F_{3} \\ H_{3}\end{array}\right)$,

where $p, q, s, t$ are integers constrained by $p t-q s=1$ and $R \in S L(2, \mathbb{Z})_{\tau}$.

Similarly, each torus $T_{i}^{2}$ has the modular symmetry $S L(2, \mathbb{Z})_{i}$ for vanishing three-form fluxes. Each torus $T_{i}^{2}=$ $R_{i}^{2} / \Lambda_{i}$ is defined by the two-dimensional Euclidean space $R_{i}^{2}$ modded out by the lattice $\Lambda_{i}$, and the lattice $\Lambda_{i}$ is spanned by the basis vectors $\left(e_{x^{i}}, e_{y^{i}}\right)$. The same lattice is spanned by other basis vectors $\left(e_{x^{i}}^{\prime}, e_{y^{i}}^{\prime}\right)$ satisfying

$$
\left(\begin{array}{c}
e_{y^{i}}^{\prime} \\
e_{x^{i}}^{\prime}
\end{array}\right)=R_{i}\left(\begin{array}{c}
e_{y^{i}} \\
e_{x^{i}}
\end{array}\right)
$$

with

$$
R_{i}=\left(\begin{array}{cc}
p_{i} & q_{i} \\
s_{i} & t_{i}
\end{array}\right) \in S L(2, \mathbb{Z})_{i}
$$

with $p_{i} t_{i}-q_{i} s_{i}=1$. This is the modular transformation, $S L(2, \mathbb{Z})_{i}$. Then, the shape of each torus $T_{i}^{2}$, i.e., the modulus parameter $\tau_{i}$ transforms,

$$
\tau_{i} \equiv \frac{e_{y^{i}}}{e_{x^{i}}} \rightarrow \tau_{i}^{\prime} \equiv \frac{e_{y^{i}}^{\prime}}{e_{x^{i}}^{\prime}}=\frac{p_{i} \tau_{i}+q_{i}}{s_{i} \tau_{i}+t_{i}}=R_{i}\left(\tau_{i}\right),
$$

under the modular transformation. It is noted that the generators of the modular symmetry are given by

$$
S: \tau_{i} \rightarrow-\frac{1}{\tau_{i}}, \quad T: \tau_{i} \rightarrow \tau_{i}+1,
$$

which satisfy $S^{2}=(S T)^{3}=1$.

When we introduce the coordinates of each torus by choosing $e_{y^{i}}=\tau_{i}$ and $e_{x^{i}}=1$,

$$
z_{i}=x_{i}+\tau_{i} y_{i}=\left(y_{i}, x_{i}\right) \cdot\left(\begin{array}{c}
\tau_{i} \\
1
\end{array}\right)
$$

it is equivalent to

$$
z_{i}^{\prime}=x_{i}^{\prime}+\tau_{i}^{\prime} y_{i}^{\prime}=\left(y_{i}, x_{i}\right) R_{i}^{-1} R_{i}\left(\begin{array}{c}
\tau_{i} \\
1
\end{array}\right)
$$

under the modular transformation $R_{i} \in S L(2, \mathbb{Z})_{i}$. Hence, the coordinates of each torus $\left(y_{i}, x_{i}\right)$ are related to $\left(y_{i}^{\prime}, x_{i}^{\prime}\right)$,

$$
\left(\begin{array}{c}
y_{i}^{\prime} \\
x_{i}^{\prime}
\end{array}\right)=\left(R_{i}^{-1}\right)^{T}\left(\begin{array}{c}
y_{i} \\
x_{i}
\end{array}\right) .
$$

It indicates that under the modular transformations of $\bigotimes_{i=1}^{3}$ $S L(2, \mathbb{Z})_{i}$ associated with three 2-tori, not only the complex structure moduli $\tau_{i}$ are related to $\tau_{i}^{\prime}$, but also the three-form fluxes $\left(F_{3}, H_{3}\right)$ in Eq. (4) nontrivially transform to $\left(F_{3}^{\prime}, H_{3}^{\prime}\right)$ because of the coordinate transformations of $\left(y_{i}, x_{i}\right)$. Recalling that the Kähler potential of the moduli $\tau_{i}$ transforms

$$
\begin{aligned}
& -\ln \left(i\left(\tau_{1}-\bar{\tau}_{1}\right)\left(\tau_{2}-\bar{\tau}_{2}\right)\left(\tau_{3}-\bar{\tau}_{3}\right)\right) \\
& \rightarrow-\ln \left(i\left(\tau_{1}-\bar{\tau}_{1}\right)\left(\tau_{2}-\bar{\tau}_{2}\right)\left(\tau_{3}-\bar{\tau}_{3}\right)\right)+\ln \left[\Pi_{i=1}^{3}\left|s_{i} \tau_{i}+t_{i}\right|^{2}\right],
\end{aligned}
$$

the effective action has a modular invariance only when the transformation of the superpotential (2) is provided by

$$
W \rightarrow \frac{W}{\Pi_{i=1}^{3}\left(s_{i} \tau_{i}+t_{i}\right)} .
$$

Note that it is enforced by the fact that the Kähler potential and the superpotential appear in the combination $K+$ $\ln |W|^{2}$ in the 4D $\mathcal{N}=1$ supergravity action. Hence, taking into account the modular transformation of the holomorphic three form,

$$
\Omega \rightarrow \frac{\Omega}{\Pi_{i=1}^{3}\left(s_{i} \tau_{i}+t_{i}\right)},
$$


$\bigotimes_{i=1}^{3} S L(2, \mathbb{Z})_{i}$ modular symmetries remain in the effective action, only when $G_{3}$ itself is invariant under the modular transformations.

\section{Congruence subgroups of the modular group}

In this section, we discuss the congruence subgroups of the modular group, which can arise by the spontaneous breaking of the modular group on the three-form flux background.

To demonstrate the realization of the congruence subgroups of the modular groups, we consider the following simplified superpotential:

$$
\begin{aligned}
W= & \tau_{3}\left[a^{0} \tau_{1} \tau_{2}-a^{1} \tau_{2}-a^{2} \tau_{1}-b_{3}\right] \\
& +\tau\left[c^{3} \tau_{1} \tau_{2}+d_{1} \tau_{1}+d_{2} \tau_{2}+d_{0}\right] \\
= & \left(\tau_{3}-f \tau\right)\left[a^{0} \tau_{1} \tau_{2}-a^{1} \tau_{2}-a^{2} \tau_{1}-b_{3}\right],
\end{aligned}
$$

where we chose the nonvanishing fluxes as

$$
c^{3}=-f a^{0}, \quad d_{1}=f a^{2}, \quad d_{2}=f a^{1}, \quad d_{0}=f b_{3},
$$

and the other fluxes vanish. The supersymmetric minimum is given by

$$
\tau_{3}=f \tau, \quad \tau_{1}=\frac{a^{1} \tau_{2}+b_{3}}{a^{0} \tau_{2}-a^{2}},
$$

on which there exist flat directions on the moduli spaces. In the following subsections, we explicitly show the possible congruence subgroups on such flat directions. Note that the above fluxes induce the D3-brane charge,

$N_{\text {flux }}=-d_{0} a^{0}+c^{3} b_{3}-d_{1} a^{1}-d_{2} a^{2}=-2 d_{0} a^{0}-2 d_{1} a^{1}$,

which is quantized in multiples of 8 on $T^{6} / \mathbb{Z}_{2}$ background due to Eq. (5).

\section{Model 1}

We first discuss the simplest case where the superpotential (21) reduces to be

$$
W=-\left(\tau_{3}-f \tau\right)\left(a^{2} \tau_{1}+a^{1} \tau_{2}\right),
$$

by further setting $a^{0}=b_{3}=0$ in (21). Note that the flux quanta $a^{1,2}$ are even integers; otherwise, we have to introduce exotic O3-plane contributions. The above form of the superpotential has already been discussed in [64]. Such a superpotential is induced by the following set of three-form fluxes:

$$
\begin{aligned}
F_{3} & =\left(a^{2} d x_{1} \wedge d y^{2}-a^{1} d y^{1} \wedge d x_{2}\right) \wedge d x_{3} \\
& \equiv A_{i j} d \xi_{1}^{i} \wedge d \xi_{2}^{j} \wedge d x_{3}, \\
H_{3} & =-f\left(a^{2} d x_{1} \wedge d y^{2}+a^{1} d y^{1} \wedge d x_{2}\right) \wedge d y_{3}, \\
& \equiv-f A_{i j} d \xi_{1}^{i} \wedge d \xi_{2}^{j} \wedge d y_{3},
\end{aligned}
$$

with

$$
A_{i j} \equiv\left(\begin{array}{cc}
0 & a^{1} \\
a^{2} & 0
\end{array}\right), \quad \xi_{i} \equiv\left(\begin{array}{c}
y_{i} \\
x_{i}
\end{array}\right) .
$$

The supersymmetric minimum $\partial_{\tau} W=\partial_{\tau_{i}} W=W=0$ with $i=1,2,3$ has two flat directions,

$$
\tau_{3}=f \tau, \quad a^{2} \tau_{1}=-a^{1} \tau_{2},
$$

from which we require $\operatorname{sign}(f)=-\operatorname{sign}\left(a^{1} a^{2}\right)=1$ to realize $\operatorname{Im}(\tau), \operatorname{Im}\left(\tau_{1,2,3}\right)>0$ in our conventions. Then, the tadpole cancellation condition (9) reads

$$
0 \leq-f a^{1} a^{2} \leq 16
$$

from which $f \neq 1$ gives the severe condition to the choice of $a^{1}$ and $a^{2}$. As discussed later, only the ratio $a^{1} / a^{2}$ is important to classify the modular subgroups in the flat direction $a^{2} \tau_{1}=-a^{1} \tau_{2}$. Thus, the tadpole cancellation condition (29) with $f \neq 1$ restricts the choice of $a^{1}$ and $a^{2}$ in comparison with the $f=1$ case, which has the least constraint on $a^{1}$ and $a^{2}$. For that reason, we restrict ourselves to the $f=1$ case in the following discussion.

We first focus on the $S L(2, \mathbb{Z})_{3}$ modular transformations of the $\left(F_{3}, H_{3}\right)$ pair provided by

$$
\left(\begin{array}{l}
F_{3}^{\prime} \\
H_{3}^{\prime}
\end{array}\right)=R_{3}\left(\begin{array}{c}
F_{3} \\
H_{3}
\end{array}\right)
$$

from which the total action is not invariant under $S L(2, \mathbb{Z})_{3}$. This is because $G_{3}$ itself transforms under the modular transformation which does not lead to the transformation of the superpotential as in (19). However, if we identify $R_{3}=$ $R$ and take $f=1$, a diagonal part of $S L(2, \mathbb{Z})_{\tau} \times S L(2, \mathbb{Z})_{3}$ remains in the effective action. Indeed, in such a case, we can verify that the vacuum condition $\tau_{3}=\tau$ still holds after the modular transformation,

$$
\tau_{3}^{\prime}=R_{3}\left(\tau_{3}\right)=R\left(\tau_{3}\right)=R(\tau)=\tau^{\prime}
$$

where we employ $R_{3}=R$ and $\tau_{3}=\tau$.

Next, let us discuss the modular transformations on $T_{1}^{2} \times T_{2}^{2}$, namely, $\tau_{1}^{\prime}=R_{1}\left(\tau_{1}\right)$ and $\tau_{2}^{\prime}=R_{2}\left(\tau_{2}\right)$, under which three-form fluxes transform 


$$
\begin{aligned}
& F_{3} \rightarrow\left(R_{1}^{-1} A\left(R_{2}^{-1}\right)^{T}\right)_{i j} d \xi_{1}^{i} \wedge d \xi_{2}^{j} \wedge d x_{3} \\
& H_{3} \rightarrow-f\left(R_{1}^{-1} A\left(R_{2}^{-1}\right)^{T}\right)_{i j} d \xi_{1}^{i} \wedge d \xi_{2}^{j} \wedge d y_{3} .
\end{aligned}
$$

To be invariant under the modular transformations, we require that

$$
R_{1}^{-1} A\left(R_{2}^{-1}\right)^{T}=A,
$$

which is possible under

$$
R_{2}=A^{T}\left(R_{1}^{-1}\right)^{T}\left(A^{-1}\right)^{T}=\left(\begin{array}{cc}
p_{1} & -q_{1} a^{2} / a^{1} \\
-s_{1} a^{1} / a^{2} & t_{1}
\end{array}\right) .
$$

It turns out that $q_{1}$ and $s_{1}$ are multiples of $a^{1}$ and $a^{2}$, respectively. Similar to $\tau_{3}=\tau$, we can check that $a^{2} \tau_{1}=$ $-a^{1} \tau_{2}$ also holds after the modular transformations,

$$
\begin{aligned}
a^{2} \tau_{1}^{\prime} & =a^{2} R_{1}\left(\tau_{1}\right)=a^{2} \frac{p_{1} \tau_{1}+q_{1}}{s_{1} \tau_{1}+t_{1}}=-a^{1} \frac{p_{1} \tau_{2}-q_{1} a^{2} / a^{1}}{-\left(s_{1} a^{1} / a^{2}\right) \tau_{2}+t_{1}} \\
& =-a^{1} R_{2}\left(\tau_{2}\right)=-a^{1} \tau_{2}^{\prime},
\end{aligned}
$$

where we employ $a^{2} \tau_{1}=-a^{1} \tau_{2}$.

Interestingly, the fundamental region of the moduli spaces reduces to the region characterized by the matrices $R_{1}$ and $R_{2}$, enumerated as follows:

(i) $\left|a^{2} / a^{1}\right|=n\left(\in \mathbb{Z}_{>0}\right)$

$$
\begin{aligned}
& R_{1}=\left(\begin{array}{cc}
1 & 1 \\
0(\bmod n) & 1
\end{array}\right) \in \Gamma_{0}(n), \\
& R_{2}=\left(\begin{array}{cc}
1 & 0(\bmod n) \\
1 & 1
\end{array}\right) \in \Gamma^{0}(n),
\end{aligned}
$$

(ii) $\left|a^{1} / a^{2}\right|=n\left(\in \mathbb{Z}_{>0}\right)$

$$
\begin{aligned}
& R_{1}=\left(\begin{array}{cc}
1 & 0(\bmod n) \\
1 & 1
\end{array}\right) \in \Gamma^{0}(n), \\
& R_{2}=\left(\begin{array}{cc}
1 & 1 \\
0(\bmod n) & 1
\end{array}\right) \in \Gamma_{0}(n),
\end{aligned}
$$

(iii) $\operatorname{gcd}\left(a^{1}, a^{2}\right)=1$

$$
\begin{aligned}
& R_{1}=\left(\begin{array}{cc}
1 & 0\left(\bmod a^{1}\right) \\
0\left(\bmod a^{2}\right) & 1
\end{array}\right) \in \Gamma\left(\left|a^{1} a^{2}\right|\right), \\
& R_{2}=\left(\begin{array}{cc}
1 & 0\left(\bmod a^{2}\right) \\
0\left(\bmod a^{1}\right) & 1
\end{array}\right) \in \Gamma\left(\left|a^{1} a^{2}\right|\right) .
\end{aligned}
$$

Several congruence subgroups are defined in the Appendix. Note that $\Gamma_{0}(1)=\Gamma^{0}(1)=\Gamma(1)=S L(2, \mathbb{Z})$. It indicates that the $S L(2, \mathbb{Z})_{1,2}$ groups are spontaneously broken to
TABLE I. Possible congruence subgroups of $\left\{S L(2, \mathbb{Z})_{1}\right.$, $\left.S L(2, \mathbb{Z})_{2}\right\}$ except for the trivial pattern $a^{1}=a^{2}$. Those are constrained by the tadpole cancellation condition (29) and quantization condition of flux quanta $a^{1}, a^{2} \in 2 \mathbb{Z}$. Here, we restrict ourselves to the case with $a^{2} / a^{1}>1$, but it is also possible to consider the case with the replacement $\Gamma^{(2)}$ of $\Gamma^{(1)}$.

\begin{tabular}{lc}
\hline \hline$f$ & $\left\{\Gamma^{(1)}, \Gamma^{(2)}\right\}$ \\
\hline 1 & $\left\{\Gamma_{0}(2), \Gamma^{0}(2)\right\},\left\{\Gamma_{0}(3), \Gamma^{0}(3)\right\},\left\{\Gamma_{0}(4), \Gamma^{0}(4)\right\}$ \\
\hline \hline
\end{tabular}

their congruence subgroups $\Gamma^{(1)}$ and $\Gamma^{(2)}$. Given that $a^{1}$ and $a^{2}$ are quantized to be even on $T^{6} / \mathbb{Z}_{2}$ background, we obtain the subgroups of $S L(2, \mathbb{Z})_{1,2}$ as shown in Table I.

In this way, the low-energy effective action has discrete modular symmetries below the mass scale of heavy modulus. The remaining modular group in the low-energy effective action is severely constrained by the quantization of fluxes and tadpole cancellation condition. We have focused on the specific moduli stabilization, but we expect that allowed congruence subgroups are restricted in a general choice of flux quanta on $T^{6} / \mathbb{Z}_{2}$ due to the quantization of three-form fluxes (5). In the following, we show other examples as the generalization of our setup.

\section{Model 2}

So far, our approach has been restricted to the superpotential (39) leading to the flat directions in the moduli spaces of the axiodilaton and complex structure moduli. ${ }^{3}$ Here the flat direction leading to the discrete modular groups can be achieved in the linear combination of two complex structure moduli fields, but it is possible to obtain more complicated flat directions of the moduli space in general. In this section, we show next nontrivial examples leading to the flat direction of the moduli space.

We consider the following superpotential [64]:

$$
W=\left(\tau_{3}-f \tau\right)\left[a^{0} \tau_{1} \tau_{2}-b_{3}\right],
$$

by further imposing $a^{1}=a^{2}=0$ in Eq. (21). We find the supersymmetric minimum

$$
\tau_{1} \tau_{2}=\frac{b_{3}}{a^{0}}, \quad \tau_{3}=f \tau,
$$

and the flux-induced D3-brane charge is given by

$$
N_{\text {flux }}=-d_{0} a^{0}+c^{3} b_{3}=-2 f a^{0} b^{3} .
$$

In this case, the three-form fluxes are introduced on the following basis:

\footnotetext{
${ }^{3}$ For the stabilization of all the complex structure moduli and axiodilaton at supersymmetric and supersymmetry-breaking minima, we refer [73].
} 


$$
\begin{aligned}
F_{3} & =\left(a^{0} d x_{1} \wedge d x^{2}-b_{3} d y^{1} \wedge d y_{2}\right) \wedge d x_{3} \\
& \equiv A_{i j} d \xi_{1}^{i} \wedge d \xi_{2}^{j} \wedge d x_{3}, \\
H_{3} & =\left(c_{3} d x_{1} \wedge d x^{2}+d_{0} d y^{1} \wedge d y_{2}\right) \wedge d y_{3}, \\
& \equiv-f A_{i j} d \xi_{1}^{i} \wedge d \xi_{2}^{j} \wedge d y_{3},
\end{aligned}
$$

with

$$
A_{i j} \equiv\left(\begin{array}{cc}
-b_{3} & 0 \\
0 & a^{0}
\end{array}\right), \quad \xi_{i} \equiv\left(\begin{array}{c}
y_{i} \\
x_{i}
\end{array}\right) .
$$

From the modular transformation of the three-form fluxes,

$$
\begin{aligned}
& F_{3} \rightarrow\left(R_{1}^{-1} A\left(R_{2}^{-1}\right)^{T}\right)_{i j} d \xi_{1}^{i} \wedge d \xi_{2}^{j} \wedge d x_{3}, \\
& H_{3} \rightarrow-f\left(R_{1}^{-1} A\left(R_{2}^{-1}\right)^{T}\right)_{i j} d \xi_{1}^{i} \wedge d \xi_{2}^{j} \wedge d y_{3},
\end{aligned}
$$

we require $R_{1}^{-1} A\left(R_{2}^{-1}\right)^{T}=A$ in a way similar to the model 1. It can be satisfied under

$R_{2}=A^{T}\left(R_{1}^{-1}\right)^{T}\left(A^{-1}\right)^{T}=\left(\begin{array}{cc}t_{1} & s_{1} b_{3} / a^{0} \\ q_{1} a^{0} / b_{3} & p_{1}\end{array}\right)$.

It turns out that $q_{1}$ and $s_{1}$ are multiples of $b_{3}$ and $a^{0}$, respectively.

We checked that the vacuum condition $\tau_{3}=\tau$ holds after the modular transformation in model 1 , where we choose $f=1$. It is straightforward to check that $\tau_{1} \tau_{2}=b_{3} / a^{0}$ also holds after the modular transformations,

$$
\begin{aligned}
\tau_{1}^{\prime} \tau_{2}^{\prime} & =R_{1}\left(\tau_{1}\right) R_{2}\left(\tau_{2}\right)=\left(\frac{p_{1} \tau_{1}+q_{1}}{s_{1} \tau_{1}+t_{1}}\right) \frac{t_{1} \tau_{2}+s_{1} b_{3} / a^{0}}{\left(q_{1} a^{0} / b_{3}\right) \tau_{2}+p_{1}} \\
& =\frac{b_{3}}{a^{0}}\left(\frac{p_{1} \tau_{1}+q_{1}}{s_{1} \tau_{1}+t_{1}}\right) \frac{t_{1} / \tau_{1}+s_{1}}{q_{1} / \tau_{1}+p_{1}}=\frac{b_{3}}{a^{0}}
\end{aligned}
$$

where we employ $\tau_{1} \tau_{2}=b_{3} / a^{0}$.

As a result, the remaining congruence subgroups are the same as in model 1, as shown in Table I, when we replace the flux pair $\left(a^{1}, a^{2}\right)$ in model 1 by the flux pair $\left(a^{0}, b^{3}\right)$. This is because the tadpole cancellation condition as well as the matrix $R_{2}$ has the same structure.

\section{Model 3}

Here, we discuss other nontrivial examples leading to the congruence subgroups in the flat direction of the moduli space. Let us consider the following superpotential:

$$
W=\left(\tau_{3}-f \tau\right)\left[a^{0} \tau_{1} \tau_{2}-a^{2} \tau_{1}-b_{3}\right],
$$

by setting $a^{1}=0$ in Eq. (21), ${ }^{4}$ indicating that the fluxinduced D3-brane charge is given by

$$
N_{\text {flux }}=-d_{0} a^{0}+c^{3} b_{3}=-2 f a^{0} b^{3} .
$$

\footnotetext{
${ }^{4}$ The following discussion is also applicable in the case with $a^{2}=0$ and $a^{1} \neq 0$.
}

The supersymmetric minimum $\partial_{\tau} W=\partial_{\tau_{i}} W=W=0$ with $i=1,2,3$ is realized at

$$
\tau_{3}=f \tau, \quad a^{0} \tau_{1} \tau_{2}-a^{2} \tau_{1}-b_{3}=0 .
$$

In a way similar to the analyses in the previous subsections, we discuss the congruence subgroups on the above flat directions of the moduli space. Since the $\tau_{3}=f \tau$ direction is the same with models 1 and 2, we focus on the other flat direction. The three-form fluxes are introduced as follows:

$$
\begin{aligned}
F_{3}= & \left(a^{0} d x_{1} \wedge d x^{2}+a^{2} d x_{1} \wedge d y^{2}-b_{3} d y^{1} \wedge d y_{2}\right) \\
& \wedge d x_{3} \equiv A_{i j} d \xi_{1}^{i} \wedge d \xi_{2}^{j} \wedge d x_{3}, \\
H_{3}= & \left(c_{3} d x_{1} \wedge d x^{2}-d_{1} d x_{1} \wedge d y^{2}+d_{0} d y^{1} \wedge d y_{2}\right) \\
& \wedge d y_{3}, \equiv-f A_{i j} d \xi_{1}^{i} \wedge d \xi_{2}^{j} \wedge d y_{3},
\end{aligned}
$$

with

$$
A_{i j} \equiv\left(\begin{array}{cc}
-b_{3} & 0 \\
a^{2} & a^{0}
\end{array}\right), \quad \xi_{i} \equiv\left(\begin{array}{c}
y_{i} \\
x_{i}
\end{array}\right) .
$$

We require $R_{1}^{-1} A\left(R_{2}^{-1}\right)^{T}=A$ to keep the invariance of the three-form fluxes under the modular transformations on $S L(2, \mathbb{Z})_{1} \times S L(2, \mathbb{Z})_{2}$. We find that it can be achieved when

$$
\begin{aligned}
R_{2} & =A^{T}\left(R_{1}^{-1}\right)^{T}\left(A^{-1}\right)^{T} \\
& =\left(\begin{array}{cc}
1 & \frac{a^{2}}{b_{3}} \frac{b_{3}}{a^{0}} \\
0 & 1
\end{array}\right)\left(\begin{array}{cc}
t_{1} & s_{1} b_{3} / a^{0} \\
q_{1} a^{0} / b_{3} & p_{1}
\end{array}\right)\left(\begin{array}{cc}
1 & -\frac{a^{2}}{b_{3}} \frac{b_{3}}{a^{0}} \\
0 & 1
\end{array}\right) .
\end{aligned}
$$

In this way, it is the generalization of (45). When $a^{2} / b_{3} \in$ $\mathbb{Z}$ and $b_{3} / a^{0} \in \mathbb{Z}$, Eq. (52) is the element of $\Gamma^{0}\left(b_{3} / a^{0}\right)$; otherwise, it is difficult to obtain the congruence subgroups. Then, $q_{1}$ and $s_{1}$ are required to be multiples of $b_{3}$ and $a^{0}$, respectively. Since the tadpole cancellation condition as well as the structure of the matrix $R_{2}$ is the same with models 1 and 2, the remaining modular group in the effective action is described by Table I. Note that it is straightforward to check the modular invariance of the vacuum condition (49) by employing Eqs. (52) and (49).

So far, we have discussed the congruence subgroups as a special case of Eq. (21) by setting certain fluxes to be 0 . As a result, flat direction in Eq. (23) has the congruence subgroups enumerated in Eqs. (36)-(38). The important point to realize the (discrete) modular groups in the effective action is that the three-form fluxes are expanded on the basis $\xi$ which manifests the modular invariance of $F_{3}$ and $H_{3}$. If the basis of $F_{3}$ and $H_{3}$ explicitly depends on the coordinates $x$ and $y\left[x_{3}, y_{3}\right.$ in the previous setup (44)], the modular transformations change the $G_{3}$ itself and $S L(2, \mathbb{Z})_{\tau}$ transformation is required to compensate the transformation of $G_{3}$ as demonstrated in $\tau_{3}=\tau$ direction (31). Hence, we expect that the flat directions possessing 
the modular symmetries in the superpotential (7) are typically characterized by the two moduli fields among $\left(\tau_{1}, \tau_{2}, \tau_{3}, S\right)$, and our discussed superpotential is a representative one. This argument will hold not only $T^{6} / \mathbb{Z}_{2}$, but also more general toroidal orientifolds as well.

Let us briefly comment on other toroidal orientifolds. Compared with the $T^{6} / \mathbb{Z}_{2}$ and $T^{6} /\left(\mathbb{Z}_{2} \times \mathbb{Z}_{2}^{\prime}\right)$ orientifolds, the untwisted complex structure moduli are fixed at discrete values or described by the single modulus on other toroidal orbifolds preserving the supersymmetry such as $T^{6} /\left(\mathbb{Z}_{2} \times \mathbb{Z}_{3}\right)=T^{6} / \mathbb{Z}_{6-I I}, \quad T^{6} /\left(\mathbb{Z}_{2} \times \mathbb{Z}_{6}\right), \quad T^{6} / \mathbb{Z}_{4}$, $T^{6} / \mathbb{Z}_{8-I I}$, and $T^{6} / \mathbb{Z}_{12-I I}$ [74-77]. The presence of modular symmetry on other toroidal orientifolds is a restricted class of $T^{6} / \mathbb{Z}_{2}$ orientifold, taking into account the tadpole cancellation conditions as well as the quantization condition of fluxes.

\section{MODULAR SYMMETRY ON $T^{6} /\left(\mathbb{Z}_{2} \times \mathbb{Z}_{2}^{\prime}\right)$ ORIENTIFOLDS WITH MAGNETIZED D-BRANES}

So far, we have not considered the matter sector. In this section, we introduce the magnetized D-branes on $T^{6} /\left(\mathbb{Z}_{2} \times \mathbb{Z}_{2}^{\prime}\right)$ orientifolds rather than $T^{6} / \mathbb{Z}_{2}$. Similar to the factorizable six-torus $T^{6} / \mathbb{Z}_{2}$ discussed in the previous section, the three forms can be expanded on the same basis in (6) which are invariant under the $\mathbb{Z}_{2} \times \mathbb{Z}_{2}^{\prime}$ orbifoldings. In this way, we can apply the moduli stabilization scheme of Sec. II, in particular model 1 in Sec. II C 1 , to $T^{6} /\left(\mathbb{Z}_{2} \times\right.$ $\left.\mathbb{Z}_{2}^{\prime}\right)$ background. It indicates that the congruence subgroups also appear in the 4D effective action, although the tadpole cancellation condition and the quantization condition of fluxes are modified due to the orientifold contributions and inclusion of the D-branes with magnetic fluxes.

\section{A. $T^{6} /\left(\mathbb{Z}_{2} \times \mathbb{Z}_{2}^{\prime}\right)$ orientifold models}

In this section, we review the $T^{6} /\left(\mathbb{Z}_{2} \times \mathbb{Z}_{2}^{\prime}\right)$ orientifold models with or without discrete torsion, where the semirealistic phenomenological models are previously found in D-branes with magnetic fluxes [65-68].

On this background, two $\mathbb{Z}_{2}$ symmetries act on the $T^{6}$ coordinates as

$$
\begin{aligned}
\theta:\left(z_{1}, z_{2}, z_{3}\right) & \rightarrow\left(-z_{1},-z_{2}, z_{3}\right), \\
\theta^{\prime}:\left(z_{1}, z_{2}, z_{3}\right) & \rightarrow\left(z_{1},-z_{2},-z_{3}\right),
\end{aligned}
$$

and the orientifold projection is characterized by the worldsheet parity projection $\Omega$ and

$$
\mathcal{R}:\left(z_{1}, z_{2}, z_{3}\right) \rightarrow\left(-z_{1},-z_{2},-z_{3}\right) .
$$

Under those actions, there exist $64 \mathrm{O} 3$ planes located at a fixed point of $\mathcal{R}$ and $4 \mathrm{O}_{1^{-}}, 4 \mathrm{O}_{2^{-}}, 4 \mathrm{O}_{3^{-}}$planes, located at the fixed locus of $\mathcal{R} \theta^{\prime}, \mathcal{R} \theta \theta^{\prime}$, and $\mathcal{R} \theta$, respectively.
In addition, it is possible to consider $N_{a}$ stacks of magnetized $\mathrm{D}(3+2 n)$ branes wrapping $2 n$ cycles on $T^{6} /\left(\mathbb{Z}_{2} \times \mathbb{Z}_{2}^{\prime}\right)$, where $U(1)_{a}$ magnetic fluxes $F_{a}$ are quantized on $T_{i}^{2}$,

$$
\frac{m_{a}^{i}}{2 \pi} \int_{T_{i}^{2}} F_{a}^{i}=n_{a}^{i}
$$

Here the integer $m_{a}^{i}$ denotes the wrapping number of $N_{a}$ $\mathrm{D}(3+2 n)$ branes around $T_{i}^{2}$ and $n_{a}^{i}$ are quantized fluxes. Note that D3, D5, D7, D9 branes can consider 0, 1, 2, and 3 nonvanishing $m_{a}^{i}$ fluxes, respectively. Under the orientifold projection, the wrapping number $m_{a}^{i}$ transforms as $\Omega \mathcal{R}: m_{a}^{i} \rightarrow-m_{a}^{i}$. Such gauge fluxes not only break the original gauge symmetry of $\mathrm{D}(3+2 n)$ branes, but also induce the chiral zero modes at the intersection of two stacks of D-branes, counted by

$$
I_{a b}=\Pi_{i=1}^{3}\left(n_{a}^{i} m_{b}^{i}-n_{b}^{i} m_{a}^{i}\right),
$$

where the labels $a$ and $b$ represent two stacks of D-branes.

Recall that the above magnetic fluxes also carry the Ramond-Ramond (RR) charges of lower-dimensional D-branes through the Chern-Simons coupling. Thanks to the orientifold projection, RR tadpoles of D5 and D9 branes are canceled by their orientifold images. It is thus required to take into account only the D3- and D7-brane charges [67],

$$
\begin{aligned}
& \mathrm{D} 3: \sum_{a} N_{a} n_{a}^{1} n_{a}^{2} n_{a}^{3}+\frac{1}{2} N_{\text {flux }}=16, \\
& \mathrm{D} 7_{1}: \sum_{a} N_{a} n_{a}^{1} m_{a}^{2} m_{a}^{3}=-16, \\
& \mathrm{D} 7_{2}: \sum_{a} N_{a} n_{a}^{2} m_{a}^{1} m_{a}^{3}=-16, \\
& \mathrm{D} 7_{3}: \sum_{a} N_{a} n_{a}^{3} m_{a}^{1} m_{a}^{2}=-16,
\end{aligned}
$$

where $\mathrm{O} 3$ and $\mathrm{O}_{i}$ planes have $-1 / 2$ units of $\mathrm{D} 3$-brane charge and -8 units of $\mathrm{D} 7_{i}$-brane charge, respectively.

Our interest is to reveal the allowable values of flux quanta $N_{\text {flux }}$, determining the breaking of the modular symmetry as discussed in the previous section. On $T^{6} /\left(\mathbb{Z}_{2} \times \mathbb{Z}_{2}^{\prime}\right)$ background, one can introduce the discrete torsion in $\mathbb{Z}_{2}$ twisted sector [78-80], which changes a part of the orientifold charges and the quantization condition of fluxes. The quantization of the three-form fluxes on $\mathbb{Z}_{2} \times \mathbb{Z}_{2}^{\prime}$ with discrete torsion, ${ }^{5}$ the three-form fluxes $F_{3}$ and $H_{3}$ are quantized in multiples of 8, due to the

\footnotetext{
${ }^{5}$ We use the conventions that the hodge numbers of $T^{6} /\left(\mathbb{Z}_{2} \times\right.$ $\left.\mathbb{Z}_{2}^{\prime}\right)$ are $\left(h_{1,1}, h_{2,1}\right)=(3,51)$ with discrete torsion and $\left(h_{1,1}, h_{2,1}\right)=(51,3)$ without discrete torsion, respectively. Furthermore, we focus on the untwisted sector on $T^{6} /\left(\mathbb{Z}_{2} \times \mathbb{Z}_{2}^{\prime}\right)$ with discrete torsion.
} 
TABLE II. Possible congruence subgroups of $\left\{S L(2, \mathbb{Z})_{1}\right.$, $\left.S L(2, \mathbb{Z})_{2}\right\}$ except for the trivial $a^{1}=a^{2}$ case, up to $N_{\text {flux }}=-2 f a^{1} a^{2}=64 \times 4$. Since $N_{\text {flux }}$ is multiples of 64 due to the flux quantization of $F_{3}$ and $H_{3}$, only $N_{\text {flux }}=64 \times 4$ gives rise to the nontrivial congruence subgroup $\Gamma^{0}(2)$ and $\Gamma_{0}(2)$.

\begin{tabular}{ll}
\hline \hline$\left(f, a^{1}, a^{2}\right)$ & $\left\{\Gamma^{(1)}, \Gamma^{(2)}\right\}$ \\
\hline$(1, \mp 8, \pm 16)$ & $\left\{\Gamma^{0}(2), \Gamma_{0}(2)\right\}$ \\
$(1, \mp 16, \pm 8)$ & $\left\{\Gamma_{0}(2), \Gamma^{0}(2)\right\}$ \\
\hline \hline
\end{tabular}

$\mathbb{Z}_{2} \times \mathbb{Z}_{2}$ orbifold and orientifold projections. The reason is that the volume of a three cycle on $T^{6}$ is divided by the corresponding cycle on $T^{6} /\left(\mathbb{Z}_{2} \times \mathbb{Z}_{2}^{\prime}\right)$ [65] and the orientifold $\mathbb{Z}_{2}$ projection further act on $T^{6}[81]$. On the other hand, in the case without discrete torsion, $F_{3}$ and $H_{3}$ are quantized in multiples of 4 . (For more details, see [65], in which the convention of discrete torsion is opposite to ours.)

\section{B. Congruence subgroups}

As mentioned before, the three-form basis on $T^{6} /\left(\mathbb{Z}_{2} \times \mathbb{Z}_{2}^{\prime}\right)$ can be expanded in terms of Eq. (6). It indicates that we can apply the moduli stabilization mechanism of Sec. II into this $T^{6} /\left(\mathbb{Z}_{2} \times \mathbb{Z}_{2}^{\prime}\right)$ orientifold background, taking into account the tadpole cancellation conditions (57). As classified later, the three-form fluxes lead to the congruence subgroups on $T_{1}^{2}$ and/or $T_{2}^{2}$. After integrating out the heavy modulus, the low-energy effective action is invariant under the remaining discrete subgroups of $S L(2, \mathbb{Z})$. In the following, we enumerate the possible congruence subgroups on $T^{6} /\left(\mathbb{Z}_{2} \times \mathbb{Z}_{2}^{\prime}\right)$ with or without discrete torsion:

(i) We first discuss $T^{6} /\left(\mathbb{Z}_{2} \times \mathbb{Z}_{2}^{\prime}\right)$ with discrete torsion. Given that $F_{3}$ and $H_{3}$ are quantized in multiples of 8, namely $a^{1}, a^{2} \in 8 \mathbb{Z}$, we obtain the class of congruence subgroups of $S L(2, \mathbb{Z})_{1} \times S L(2, \mathbb{Z})_{2}$ modular group on $T_{1}^{2} \times T_{2}^{2}$, displayed in Table II. Here, we employ the model 1 in Sec. II C 1. When both the quark and lepton sectors arise in D-branes wrapping $T_{1}^{2}\left(T_{2}^{2}\right)$, their flavor symmetries are governed by the same $\Gamma^{(1)}\left(\Gamma^{(2)}\right)$. On the other hand, when their flavor symmetries are originated from different tori, for instance, the quark sector on $T_{1}^{2}$ and the lepton sector on $T_{2}^{2}$, quarks and leptons have the different flavor symmetries $\Gamma^{(1)}$ and $\Gamma^{(2)}$ as shown in Table II, respectively. Thus, our results would be interesting for bottom-up model building studied in Refs. [31,37].

(ii) Next, we focus on the case without discrete torsion, in which flux quanta $a^{1}$ and $a^{2}$ are quantized in multiples of 4 , namely, $a^{1}, a^{2} \in 4 \mathbb{Z}$. In a similar way, the flavor symmetries on D-branes wrapping $T_{1}^{2}$ and/or $T_{2}^{2}$ are governed by the congruence subgroups displayed in Table III. We have more varieties than congruence groups in Tables I and II. We can consider the same or different flavor symmetries of quark and lepton sectors, depending on the D-brane configurations.

As a result, on $T^{6} /\left(\mathbb{Z}_{2} \times \mathbb{Z}_{2}^{\prime}\right)$ orientifold background, the congruence subgroups of the modular group in the lowenergy effective action is severely constrained by the flux quantization and tadpole cancellation conditions.

\section{Concrete models}

\section{Model with discrete torsion}

In this section, we search for a concrete three-generation model including the discrete modular symmetry in a specific D-brane configuration on the $T^{6} /\left(\mathbb{Z}_{2} \times \mathbb{Z}_{2}^{\prime}\right)$ orientifold background with discrete torsion, following [67].

Massless spectrum on the stack $a$ of magnetized D-branes has the representation of $U\left(N_{a} / 2\right)$ not $U\left(N_{a}\right)$ due to the orbifold projection, meaning that $N_{a}$ must be even. There exist $\mathcal{N}=1 U\left(N_{a} / 2\right)$ vector multiplet and three chiral multiplets (open string moduli), which we refer as a $(a a)$ sector. By taking into account the chiral spectrum between two stacks $a$ and $b$ of D-branes, the massless spectrum for magnetized D-branes is summarized as follows:

$$
\begin{gathered}
a a \text { sector : }\left\{\begin{array}{c}
U\left(N_{a} / 2\right) \text { vector multiplet } \\
3 \text { Adj. chiral multiplets }
\end{array}\right. \\
a b+b a \text { sector : } I_{a b}\left(\square_{a}, \bar{\square}_{b}\right) \text { chiral multiplets, } \\
a b^{\prime}+b^{\prime} a \text { sector : } I_{a b^{\prime}}\left(\square_{a}, \square_{b}\right) \text { chiral multiplets, } \\
a a^{\prime}+a^{\prime} a \text { sector : }\left\{\begin{array}{c}
\frac{1}{2}\left(I_{a a^{\prime}}-4 I_{a, O}\right) \square \text { chiral multiplets } \\
\frac{1}{2}\left(I_{a a^{\prime}}-4 I_{a, O}\right) \boxminus \text { chiral multiplets }
\end{array}\right.
\end{gathered}
$$

where $a^{\prime}$ denotes the $\Omega \mathcal{R}$ image of the stack $a$ of D-brane and $I_{a, O}$ represents for the intersection product between the homology class of D-branes and orientifold plane.

TABLE III. Possible congruence subgroups of $\left\{S L(2, \mathbb{Z})_{1}, S L(2, \mathbb{Z})_{2}\right\}$ except for the trivial $a^{1}=a^{2}$ case, up to $N_{\text {flux }}=-2 f a^{1} a^{2}=16 \times 12$, where $N_{\text {flux }}$ is multiples of 16 due to the flux quantization of $F_{3}$ and $H_{3}$. Here, we restrict ourselves to the $\left|a^{2}\right|>\left|a^{1}\right|$ case, but it is also possible to consider the $\left|a^{1}\right|>\left|a^{2}\right|$ case.

$\frac{\left\{\Gamma^{(1)}, \Gamma^{(2)}\right\}}{\overline{\left\{\Gamma_{0}(2), \Gamma^{0}(2)\right\},\left\{\Gamma_{0}(3), \Gamma^{0}(3)\right\},\left\{\Gamma_{0}(4), \Gamma^{0}(4)\right\},\left\{\Gamma_{0}(5), \Gamma^{0}(5)\right\},\left\{\Gamma_{0}(6), \Gamma^{0}(6)\right\},\{\Gamma(6), \Gamma(6)\}}}$


TABLE IV. D-brane configurations leading to Pati-Salam model where the magnitude of magnetic fluxes $g$ corresponds to the generations chiral multiplets in the visible sector. Here, we use the identification $U S p(2) \simeq S U(2)$.

\begin{tabular}{lcccc}
\hline \hline$N_{\alpha}$ & Gauge group & $\left(n_{\alpha}^{1}, m_{\alpha}^{1}\right)$ & $\left(n_{\alpha}^{2}, m_{\alpha}^{2}\right)$ & $\left(n_{\alpha}^{3}, m_{\alpha}^{3}\right)$ \\
\hline$N_{a}=8$ & $U(4)_{a}$ & $(1,0)$ & $(g, 1)$ & $(g,-1)$ \\
$N_{b}=2$ & $S U(2)_{L}$ & $(0,1)$ & $(1,0)$ & $(0,-1)$ \\
$N_{c}=2$ & $S U(2)_{R}$ & $(0,1)$ & $(0,-1)$ & $(1,0)$ \\
$N_{h_{1}}=2$ & $U(1)_{h_{1}}$ & $(-2,-1)$ & $(3,1)$ & $(4,1)$ \\
$N_{h_{2}}=2$ & $U(1)_{h_{2}}$ & $(-2,-1)$ & $(4,1)$ & $(3,1)$ \\
$8 N_{f}$ & $U S p\left(8 N_{f}\right)$ & $(1,0)$ & $(1,0)$ & $(1,0)$ \\
\hline \hline
\end{tabular}

In addition, it is also possible to consider D-branes whose homology class is invariant under the orientifold projection for the configuration of $\mathrm{D} 3$ and $\mathrm{D} 7_{i}$ branes without magnetic fluxes. In contrast to the case without fixed by the orientifold action, the Chan-Paton gauge group for a stack of $2 N_{a}$ D-branes is described by $\operatorname{USp}\left(N_{a}\right)$ gauge group. The massless spectrum is summarized as follows:

$$
\begin{array}{r}
a a \text { sector : }\left\{\begin{array}{c}
U S p\left(N_{a}\right) \text { vector multiplet } \\
3 \boxminus \text { chiral multiplets }
\end{array},\right. \\
a b+b a \text { sector : } I_{a b}\left(\square_{a}, \bar{\square}_{b}\right) \text { chiral multiplets. }
\end{array}
$$

Let us engineer the brane configurations leading to the semirealistic model accommodating the Standard Model. The visible sector we consider consists of stacks of magnetized $\mathrm{D} 7_{a}$ with gauge group $U(4)_{a}$ and two stacks of unmagnetized $\mathrm{D} 7_{b}$ and $\mathrm{D} 7_{c}$ branes with $S U(2)_{L} \times$ $S U(2)_{R}$ as shown in Table IV. To cancel RR tadpoles, we also introduce two stacks of magnetized D9 branes with $U(1)_{h_{1}} \times U(1)_{h_{2}}$ and the $8 N_{f}$ D3 branes with $\operatorname{USp}\left(8 N_{f}\right)$ which are located at the top of an orientifold singularity.

By taking into account the Green-Schwarz mechanism, cancelling the gauge and gravitational anomalies, two $U(1)$ gauge bosons become massive at a compactification scale. Hence, remaining gauge symmetry consists of the PatiSalam and hidden sectors,

$S U(4) \times S U(2)_{L} \times S U(2)_{R} \times U(1)^{\prime} \times U S p\left(8 N_{f}\right)$, with $U(1)^{\prime}=U(1)_{a}-2 g\left(U(1)_{h_{1}}-U(1)_{h_{2}}\right)$. The latter two are the hidden sector. After all, cancellation condition of D3-brane charge (57) requires that

$$
g^{2}+N_{f}+\frac{N_{\text {flux }}}{16}=14
$$

When we focus on the three-generation model, i.e., $g=3$, we have two options to satisfy the above, namely, $\left\{N_{\text {flux }}=0, N_{f}=5\right\}$ or $\left\{N_{\text {flux }}=64, N_{f}=1\right\}$. Vanishing three-form fluxes $N_{\text {flux }}=0$ correspond to the T-dual type IIA intersecting D-brane model [82].

As demonstrated in the previous subsection, it is possible to break the modular group to its subgroup on the nontrivial three-form background. However, in the $N_{\text {flux }}=64$ case, we cannot apply our moduli stabilization scheme to this case due to $N_{\text {flux }}=-2 f a^{1} a^{2} \in 128 \mathbb{Z}$ with $a^{1}, a^{2} \in 8 \mathbb{Z}$ and $f \in \mathbb{Z}$. In the next section, we explore the possibility to realize the congruence subgroup in the toroidal orientifold model without discrete torsion.

\section{Model without discrete torsion}

The purpose of this section is to explore the existence of the congruence subgroups on $T^{6} /\left(\mathbb{Z}_{2} \times \mathbb{Z}_{2}^{\prime}\right)$ orientifold models without discrete torsion. For our purpose, we consider the specific semirealistic models, developed in Ref. [83].

Along the line of Ref. [83], we choose exotic $64 \mathrm{O} 3^{(+,+)}$ planes with positive charge and tension located at the fixed points of orbifold and orientifold actions. The $4 \mathrm{O}_{i}$ planes are located at the fixed locus of $\mathcal{R} \theta^{\prime}, \mathcal{R} \theta \theta^{\prime}$, and $\mathcal{R} \theta$, in the same way as the model with discrete torsion. Such exotic O3-plane contributions change the tadpole cancellation condition of D3-brane charge,

$$
\sum_{a} N_{a} n_{a}^{1} n_{a}^{2} n_{a}^{3}+\frac{1}{2} N_{\text {flux }}=-16,
$$

whereas the cancellation condition of $\mathrm{D} 7$-brane charges is the same with Eq. (57), taking into account the extra K-theory constraints. To demonstrate the semirealistic models, we consider the magnetic fluxes and wrapping numbers of $\mathrm{D}(3+2 n)$ branes in Table $\mathrm{V}$, where the visible sector is constructed on fractional D7 branes and D9-D99 pairs. In particular, the visible sector consists of

TABLE V. D-brane configurations leading to Pati-Salam model.

\begin{tabular}{lccccc}
\hline \hline & $N_{\alpha}$ & Gauge group & $\left(n_{\alpha}^{1}, m_{\alpha}^{1}\right)$ & $\left(n_{\alpha}^{2}, m_{\alpha}^{2}\right)$ & $\left(n_{\alpha}^{3}, m_{\alpha}^{3}\right)$ \\
\hline Fractional D-branes (visible sector) & $N_{a_{1}}=4$ & $U(4)_{C}$ & $(1,0)$ & $(0,1)$ & $(0,-1)$ \\
& $N_{a_{2}}=2$ & $U(2)_{L}$ & $(1,0)$ & $(2,1)$ & $(4,-1)$ \\
& $N_{a_{3}}=2$ & $U(2)_{R}$ & $(-3,2)$ & $(-2,1)$ & $(-4,1)$ \\
Bulk D-branes & $N_{b}=4$ & $U(2)^{2}$ & $(1,0)$ & $(0,1)$ & $(0,-1)$ \\
& $N_{c}=8$ & $U(4)^{2}$ & $(0,1)$ & $(1,0)$ & $(0,-1)$ \\
& $N_{d}=8 N_{f}$ & $U S p\left(4 N_{f}\right)^{4}$ & $(1,0)$ & $(1,0)$ & $(1,0)$ \\
\hline \hline
\end{tabular}


four-generation $\mathcal{N}=1$ supersymmetric Pati-Salam-like model with $S U(4)_{C} \times S U(2)_{L} \times S U(2)_{R}$ gauge groups, where some of the $U(1)$ gauge bosons become massive through the Green-Schwarz mechanism. For more details on the model, see Ref. [83].

Let us classify the congruence subgroups on this setup by introducing the three-form fluxes as in Sec. III C 1. From the wrapping numbers in Table V, the tadpole cancellation condition of D3-brane charges reads

$$
N_{f}+\frac{N_{\text {flux }}}{16}=2 .
$$

Here, $N_{\text {flux }}$ is quantized in multiples of 16 , due to the quantization condition of $F_{3}$ and $H_{3}$. We have three options to satisfy the above, namely, $\left\{N_{\text {flux }}=0\right.$, $\left.N_{f}=2\right\},\left\{N_{\text {flux }}=16, N_{f}=1\right\}$ and $\left\{N_{\text {flux }}=32, N_{f}=0\right\}$. The stabilization mechanism in Sec. II C 1 leads to $N_{\text {flux }}=$ $-2 f a^{1} a^{2}$ with $a^{1}, a^{2} \in 4 \mathbb{Z}$, indicating that only $N_{\text {flux }}=32$ is consistent with the tadpole cancellation condition. In that case, we have the unique possibility $\left|a^{1}\right|=\left|a^{2}\right|=4$ and $f=1$. Thus, the diagonal parts of $S L(2, \mathbb{Z})_{1} \times S L(2, \mathbb{Z})_{2}$ and $S L(2, \mathbb{Z})_{3} \times S L(2, \mathbb{Z})_{\tau}$ remain in the effective action.

In this specific Pati-Salam model having a quark-lepton unification, flavor structures of quarks and leptons are determined by the magnetic fluxes inserted on $T_{2}^{2} \times T_{3}^{2}$, indicating that both quarks and leptons transform under the same modular symmetry of $T_{2}^{2} \times T_{3}^{2}$. As a result, their flavor symmetries on $T_{2}^{2}$ could be determined by $\operatorname{SL}(2, \mathbb{Z})$.

Although we have focused on the specific D-brane configurations yielding the modular group in the effective action, it is interesting to explore D-brane models accommodating not only the three-generation models, but also the finite modular symmetry shown in Table III.

\section{CONCLUSIONS AND DISCUSSIONS}

In this paper, we have classified possible congruence subgroups of the modular group in the effective action of type IIB string theory on toroidal orientifolds with threeform fluxes. The discrete modular symmetry arises in the flat direction of complex structure moduli whose moduli space has a congruence subgroup rather than $S L(2, \mathbb{Z})$. The realization of discrete modular group in the effective action has been achieved by an existence of three-form fluxes inserted on three cycles of $T^{6}$. We argued that such a discrete modular group plays an important role of not only enlarging the axionic field range discussed in the context of swampland conjecture [64], but also the flavor symmetry of quarks and leptons.

Indeed, when magnetized D-branes wrap a certain cycle of tori, we could identify the remaining discrete modular symmetry with the flavor symmetry of quarks and/or leptons. We discussed the possible congruence subgroups on $T^{6} /\left(\mathbb{Z}_{2} \times \mathbb{Z}_{2}^{\prime}\right)$ orientifold with and without discrete torsion, incorporating the standard model sector. It turned out that the possible class of congruence subgroups are sensitive to the quantization of fluxes and the tadpole cancellation conditions. We expect that our analysis would be applicable to more broad class of D-brane model building on toroidal orientifolds. It is interesting to clarify the congruence subgroups of modular symmetry on other toroidal orientifolds (discussed in, e.g., Ref. [84]) as well as nonfactorizable tori incorporating $S L(4, \mathbb{Z})$ or $S L(6, \mathbb{Z})$ using the method in this paper. ${ }^{6}$ In this paper, we focused on type IIB flux compactifications, but our discussion is also applicable to the type IIA and heterotic string flux compactifications, taking into account the similar flux superpotential (21) and corresponding tadpole cancellation conditions. Furthermore, it would be also connected with the supergravity models having the no-scale property [87], where the moduli spaces are described by coset spaces such as $S U(p, q) /(U(1) \times S U(p) \times S U(q))$ and $S O(2,2+p) /$ $(S O(2) \times S O(2+p))$. Note that the no-scale property holds for not only the complex structure moduli in the large complex-structure limit, but also the Kähler moduli in the large-volume limit.

We have studied the geometrical symmetry, which is the full symmetry in 4D low-energy effective supergravity including closed string modes. On the other hand, matter zero modes corresponding to quarks and leptons are originated from open string modes. The number of matter zero modes is finite, and they transform under the congruence subgroups of the modular symmetry, which we have studied. Such a finite number of zero modes represent the remaining modular symmetries, which can be finite subgroups. For example, in Ref. [16], it was shown that modular symmetries of matter zero modes are discrete and finite among $S L(2, \mathbb{Z})$ in compactification with magnetic flux. They depend on the magnitude of magnetic fluxes. It is very important to study such modular symmetries of zero modes starting with our models, where the full modular symmetry is congruence subgroups. Depending on the magnitude of magnetic fluxes, we may obtain various finite modular subgroups different from those in Ref. [16]. That would provide new insights into phenomenological models with discrete flavor symmetry. We will study these issues elsewhere.

\section{ACKNOWLEDGMENTS}

T. K. was supported in part by MEXT KAKENHI Grant No. JP19H04605. H. O. was supported in part by JSPS KAKENHI Grants No. JP19J00664 and No. JP20K14477.

\section{APPENDIX: CONGRUENCE SUBGROUPS}

In this appendix, we show the conventions of the modular groups we employed. The principal congruence subgroup of level $N \in \mathbb{Z}^{+}$is

\footnotetext{
${ }^{6}$ Nonfactorizable magnetic fluxes $[85,86]$ would also be interesting.
} 


$$
\Gamma(N)=\left\{\left[\begin{array}{ll}
a & b \\
c & d
\end{array}\right] \in S L(2, \mathbb{Z}) \mid\left[\begin{array}{ll}
a & b \\
c & d
\end{array}\right] \equiv\left[\begin{array}{ll}
1(\bmod N) & 0(\bmod N) \\
0(\bmod N) & 1(\bmod N)
\end{array}\right]\right\}
$$

Definition: A subgroup $\Gamma$ of $S L(2, \mathbb{Z})$ is a congruence subgroup if $\Gamma(N) \subset \Gamma$ for some $N \in \mathbb{Z}^{+}$, in which case $\Gamma$ is a congruence subgroup of level $N$.

The congruence subgroups of Hecke type are

$$
\begin{aligned}
& \Gamma^{0}(N)=\left\{\left[\begin{array}{ll}
a & b \\
c & d
\end{array}\right] \in S L(2, \mathbb{Z}) \mid\left[\begin{array}{ll}
a & b \\
c & d
\end{array}\right] \equiv\left[\begin{array}{cc}
* & 0(\bmod N) \\
* & *
\end{array}\right]\right\}, \\
& \Gamma_{0}(N)=\left\{\left[\begin{array}{ll}
a & b \\
c & d
\end{array}\right] \in S L(2, \mathbb{Z}) \mid\left[\begin{array}{ll}
a & b \\
c & d
\end{array}\right] \equiv\left[\begin{array}{cc}
* & * \\
0(\bmod N) & *
\end{array}\right]\right\}, \\
& \Gamma_{1}(N)=\left\{\left[\begin{array}{ll}
a & b \\
c & d
\end{array}\right] \in S L(2, \mathbb{Z}) \mid\left[\begin{array}{ll}
a & b \\
c & d
\end{array}\right] \equiv\left[\begin{array}{cc}
1(\bmod N) & * \\
0(\bmod N) & 1(\bmod N)
\end{array}\right]\right\} .
\end{aligned}
$$

Note that

$$
\Gamma(1)=\Gamma_{1}(1)=\Gamma_{0}(1)=\Gamma^{0}(1)=S L(2, \mathbb{Z}) .
$$

[1] R. D. Peccei and H. R. Quinn, Phys. Rev. Lett. 38, 1440 (1977).

[2] P. W. Graham, D. E. Kaplan, and S. Rajendran, Phys. Rev. Lett. 115, 221801 (2015).

[3] J. Preskill, M. B. Wise, and F. Wilczek, Phys. Lett. 120B, 127 (1983).

[4] L. F. Abbott and P. Sikivie, Phys. Lett. 120B, 133 (1983).

[5] M. Dine and W. Fischler, Phys. Lett. 120B, 137 (1983).

[6] K. Freese, J. A. Frieman, and A. V. Olinto, Phys. Rev. Lett. 65, 3233 (1990).

[7] E. Silverstein and A. Westphal, Phys. Rev. D 78, 106003 (2008).

[8] L. McAllister, E. Silverstein, and A. Westphal, Phys. Rev. D 82, 046003 (2010).

[9] S. Ferrara, N. Magnoli, T. R. Taylor, and G. Veneziano, Phys. Lett. B 245, 409 (1990).

[10] M. Cvetic, A. Font, L. E. Ibanez, D. Lust, and F. Quevedo, Nucl. Phys. B361, 194 (1991).

[11] T. Kobayashi, D. Nitta, and Y. Urakawa, J. Cosmol. Astropart. Phys. 08 (2016) 014.

[12] S. Hamidi and C. Vafa, Nucl. Phys. B279, 465 (1987); L. J. Dixon, D. Friedan, E. J. Martinec, and S. H. Shenker, Nucl. Phys. B282, 13 (1987); T. T. Burwick, R. K. Kaiser, and H. F. Muller, Nucl. Phys. B355, 689 (1991); J. Erler, D. Jungnickel, M. Spalinski, and S. Stieberger, Nucl. Phys. B397, 379 (1993); K. S. Choi and T. Kobayashi, Nucl. Phys. B797, 295 (2008).

[13] M. Cvetic and I. Papadimitriou, Phys. Rev. D 68, 046001 (2003); S. A. Abel and A. W. Owen, Nucl. Phys. B663, 197 (2003); D. Cremades, L.E. Ibanez, and F. Marchesano,
J. High Energy Phys. 07 (2003) 038; S. A. Abel and A. W. Owen, Nucl. Phys. B682, 183 (2004).

[14] D. Cremades, L. E. Ibanez, and F. Marchesano, J. High Energy Phys. 05 (2004) 079; H. Abe, K. S. Choi, T. Kobayashi, and H. Ohki, J. High Energy Phys. 06 (2009) 080.

[15] T. Kobayashi and S. Nagamoto, Phys. Rev. D 96, 096011 (2017).

[16] T. Kobayashi, S. Nagamoto, S. Takada, S. Tamba, and T. H. Tatsuishi, Phys. Rev. D 97, 116002 (2018).

[17] T. Kobayashi, S. Nagamoto, and S. Uemura, Prog. Theor. Exp. Phys. (2017), 023B02.

[18] T. Kobayashi and S. Tamba, Phys. Rev. D 99, 046001 (2019).

[19] Y. Kariyazono, T. Kobayashi, S. Takada, S. Tamba, and H. Uchida, Phys. Rev. D 100, 045014 (2019).

[20] A. Baur, H. P. Nilles, A. Trautner, and P. K. S. Vaudrevange, Phys. Lett. B 795, 7 (2019); Nucl. Phys. B947, 114737 (2019).

[21] H. P. Nilles, S. Ramos-Sanchez, and P. K. S. Vaudrevange, J. High Energy Phys. 02 (2020) 045.

[22] F. Feruglio, arXiv:1706.08749.

[23] T. Kobayashi, K. Tanaka, and T. H. Tatsuishi, Phys. Rev. D 98, 016004 (2018).

[24] J. T. Penedo and S. T. Petcov, Nucl. Phys. B939, 292 (2019).

[25] J. C. Criado and F. Feruglio, SciPost Phys. 5, 042 (2018).

[26] T. Kobayashi, N. Omoto, Y. Shimizu, K. Takagi, M. Tanimoto, and T. H. Tatsuishi, J. High Energy Phys. 11 (2018) 196.

[27] P. P. Novichkov, J. T. Penedo, S. T. Petcov, and A. V. Titov, J. High Energy Phys. 04 (2019) 005 
[28] P. P. Novichkov, J. T. Penedo, S. T. Petcov, and A. V. Titov, J. High Energy Phys. 04 (2019) 174.

[29] F. J. de Anda, S. F. King, and E. Perdomo, Phys. Rev. D 101, 015028 (2020).

[30] H. Okada and M. Tanimoto, Phys. Lett. B 791, 54 (2019).

[31] T. Kobayashi, Y. Shimizu, K. Takagi, M. Tanimoto, T. H. Tatsuishi, and H. Uchida, Phys. Lett. B 794, 114 (2019).

[32] P. P. Novichkov, S. T. Petcov, and M. Tanimoto, Phys. Lett. B 793, 247 (2019).

[33] G. J. Ding, S. F. King, and X. G. Liu, Phys. Rev. D 100, 115005 (2019).

[34] T. Nomura and H. Okada, Phys. Lett. B 797, 134799 (2019).

[35] P. P. Novichkov, J. T. Penedo, S. T. Petcov, and A. V. Titov, J. High Energy Phys. 07 (2019) 165.

[36] H. Okada and M. Tanimoto, arXiv:1905.13421.

[37] I. De Medeiros Varzielas, S. F. King, and Y. L. Zhou, Phys. Rev. D 101, 055033 (2020).

[38] T. Nomura and H. Okada, arXiv:1906.03927.

[39] T. Kobayashi, Y. Shimizu, K. Takagi, M. Tanimoto, and T. H. Tatsuishi, arXiv:1906.10341.

[40] X. G. Liu and G. J. Ding, J. High Energy Phys. 08 (2019) 134.

[41] H. Okada and Y. Orikasa, Phys. Rev. D 100, 115037 (2019).

[42] T. Kobayashi, Y. Shimizu, K. Takagi, M. Tanimoto, and T. H. Tatsuishi, J. High Energy Phys. 02 (2020) 097.

[43] G. J. Ding, S. F. King, and X. G. Liu, J. High Energy Phys. 09 (2019) 074.

[44] H. Okada and Y. Orikasa, arXiv:1907.13520.

[45] S. F. King and Y.L. Zhou, Phys. Rev. D 101, 015001 (2020).

[46] T. Nomura, H. Okada, and O. Popov, Phys. Lett. B 803, 135294 (2020).

[47] H. Okada and Y. Orikasa, arXiv:1908.08409.

[48] J. C. Criado, F. Feruglio, F. Feruglio, and S. J. D. King, J. High Energy Phys. 02 (2020) 001.

[49] T. Kobayashi, Y. Shimizu, K. Takagi, M. Tanimoto, and T. H. Tatsuishi, Phys. Rev. D 100, 115045 (2019).

[50] T. Asaka, Y. Heo, T. H. Tatsuishi, and T. Yoshida, J. High Energy Phys. 01 (2020) 144.

[51] G. J. Ding, S. F. King, X. G. Liu, and J. N. Lu, J. High Energy Phys. 12 (2019) 030.

[52] D. Zhang, Nucl. Phys. B952, 114935 (2020).

[53] X. Wang and S. Zhou, arXiv:1910.09473.

[54] T. Kobayashi, Y. Shimizu, K. Takagi, M. Tanimoto, T. H. Tatsuishi, and H. Uchida, Phys. Rev. D 101, 055046 (2020).

[55] T. Nomura, H. Okada, and S. Patra, arXiv:1912.00379.

[56] T. Kobayashi, T. Nomura, and T. Shimomura, arXiv: 1912.00637.

[57] J. N. Lu, X. G. Liu, and G. J. Ding, arXiv:1912.07573.

[58] X. Wang, arXiv:1912.13284.
[59] R. de Adelhart Toorop, F. Feruglio, and C. Hagedorn, Nucl. Phys. B858, 437 (2012).

[60] S. B. Giddings, S. Kachru, and J. Polchinski, Phys. Rev. D 66, 106006 (2002).

[61] C. Vafa, arXiv:hep-th/0509212.

[62] H. Ooguri and C. Vafa, Nucl. Phys. B766, 21 (2007).

[63] E. Palti, Fortschr. Phys. 67, 1900037 (2019).

[64] A. Hebecker, P. Henkenjohann, and L. T. Witkowski, J. High Energy Phys. 12 (2017) 033.

[65] R. Blumenhagen, D. Lust, and T. R. Taylor, Nucl. Phys. B663, 319 (2003).

[66] J. F. G. Cascales and A. M. Uranga, J. High Energy Phys. 05 (2003) 011.

[67] F. Marchesano and G. Shiu, J. High Energy Phys. 11 (2004) 041.

[68] M. Cvetic, T. Li, and T. Liu, Phys. Rev. D 71, 106008 (2005).

[69] S. Kachru, M. B. Schulz, and S. Trivedi, J. High Energy Phys. 10 (2003) 007.

[70] S. Gukov, C. Vafa, and E. Witten, Nucl. Phys. B584, 69 (2000); B608, 477(E) (2001).

[71] A. Hanany and B. Kol, J. High Energy Phys. 06 (2000) 013.

[72] E. Witten, J. High Energy Phys. 02 (1998) 006.

[73] Y. Honma and H. Otsuka, J. High Energy Phys. 04 (2020) 001.

[74] L. E. Ibanez, J. Mas, H. P. Nilles, and F. Quevedo, Nucl. Phys. B301, 157 (1988).

[75] A. Font, L. E. Ibanez, and F. Quevedo, Phys. Lett. B 217, 272 (1989).

[76] Y. Katsuki, Y. Kawamura, T. Kobayashi, N. Ohtsubo, Y. Ono, and K. Tanioka, Nucl. Phys. B341, 611 (1990).

[77] T. Kobayashi and N. Ohtsubo, Int. J. Mod. Phys. A 09, 87 (1994).

[78] C. Vafa, Nucl. Phys. B273, 592 (1986).

[79] C. Vafa and E. Witten, J. Geom. Phys. 15, 189 (1995).

[80] M. R. Douglas, arXiv:hep-th/9807235.

[81] A. R. Frey and J. Polchinski, Phys. Rev. D 65, 126009 (2002).

[82] D. Cremades, L. E. Ibanez, and F. Marchesano, J. High Energy Phys. 07 (2003) 038.

[83] R. Blumenhagen, M. Cvetic, F. Marchesano, and G. Shiu, J. High Energy Phys. 03 (2005) 050.

[84] D. Lust, S. Reffert, W. Schulgin, and S. Stieberger, Nucl. Phys. B766, 68 (2007).

[85] I. Antoniadis, A. Kumar, and B. Panda, Nucl. Phys. B823, 116 (2009).

[86] H. Abe, T. Kobayashi, H. Ohki, K. Sumita, and Y. Tatsuta, J. High Energy Phys. 06 (2014) 017.

[87] E. Cremmer, S. Ferrara, C. Kounnas, and D. V. Nanopoulos, Phys. Lett. 133B, 61 (1983). 